\title{
Research on Planning Strategy of Power Distribution Network Integrated Energy Collaborative Optimization Based on Big Data
}

\author{
Weidong Zeng ${ }^{1}$, Zhikeng $\mathrm{Li}^{2}$,Hao Yang, Zhou Shu and Wei Liao ${ }^{1}$ \\ 1 Shenzhen Power Supply Co, Ltd 518000 \\ 2 China Energy Engineering Group Guangdong Electric Power Design Institute Co. LTD.510000
}

Keywords: Big data; Power distribution network; Energy collaborative optimization

\begin{abstract}
Power distribution network planning is the first step in the development and construction of intelligent power distribution network. Traditional power distribution network planning system and method cannot adapt to the new requirements in "big data" and "Internet plus" era, so it is urgent to speed up the construction of power distribution network planning platform. This paper analyzes the functional localization, functional requirement and data requirement of electric power enterprise for power distribution network planning intelligent assistant decision-making platform, introduces the implementation scheme of building a power distribution network integrated energy collaborative optimization based on big data based on cloud platform of electric power enterprise, including the function architecture, information architecture, data support, system integration and deployment plan. The construction of building a power distribution network integrated energy collaborative optimization auxiliary platform based on big data has important research significance and application value to improve the planning design and operation management level of intelligent power distribution network.
\end{abstract}

\section{Introduction}

With the rapid development of the economy, the energy Internet industry is rising rapidly, the intelligent power equipment is highly popularized, the randomness of the supply side is enhancing and the controllability is reducing, the demand side demands more efficient and cleaner, the volatility of demand side is increasing and the security risk of the system is increasing and the integrity is highlighting. The integrated energy system can effectively improve the efficiency of energy utilization, promote the scale development of renewable energy, and improve the consumptive ability of renewable energy while meeting the demand of pluralistic energy in the system. It is of great significance to build a physical platform with an integrated energy system as the center and organic coupling and comprehensive utilization of various energy systems. Considering the actual situation of regional development, through multi energy complementary microgrid to achieve deep coupling of energy development "source - net - charge - storage" and energy interaction and cascade utilization, the industrial park will be built into a green, environmental, energy-saving, advanced intelligent energy demonstration area, which can become a "green engine" for urban development.

\section{Demand Analysis of Power Distribution Network Integrated Energy Collaborative Optimization Platform}

Planning Strategy of Integrated Energy Collaborative Development. The energy Internet collaborative development system refers to the regional energy balance system, which takes the power system as the center and with the help of large data platform and information equipment, to realize the high coordination of each link of electricity, gas, heat, renewable energy and other "multi energy complementarity" and "source network load storage". The energy structure based on the planning area can improve the energy utilization, optimize the industrial structure and energy consumption structure, build an integrated energy station with different scale and meet the diversified energy demand. Building an energy information management platform, through the park's intelligent building and measurement information system to monitor the energy demand in 
real time and use advanced communication technology to realize the sharing of information. The energy Internet business operation mode is developed to provide users with flexible energy services, which can achieve the goal of efficient energy utilization and energy saving green development.

Model framework of integrated energy collaborative optimization. Considering the various forms of resources in the energy supply side and demand side should be integrated into a whole to make overall utilization to achieve a variety of forms of energy supply allocation based on integrated energy collaborative development. Through the construction of energy supply, energy transmission system, energy terminal and energy information system platform, fully considering the distributed energy storage system in the new area and based on data center and energy information sharing platform to achieve the multi energy complementarity coordinated development mode in the new area and coordinated planning of multi energy sources such as heat, electricity, cooling, gas and water, so as to achieve the goal of flexible supply and demand access, information coordination and interaction, energy saving and emission reduction and green environmental protection. The basic model framework of integrated energy collaborative development is shown in Figure 1.

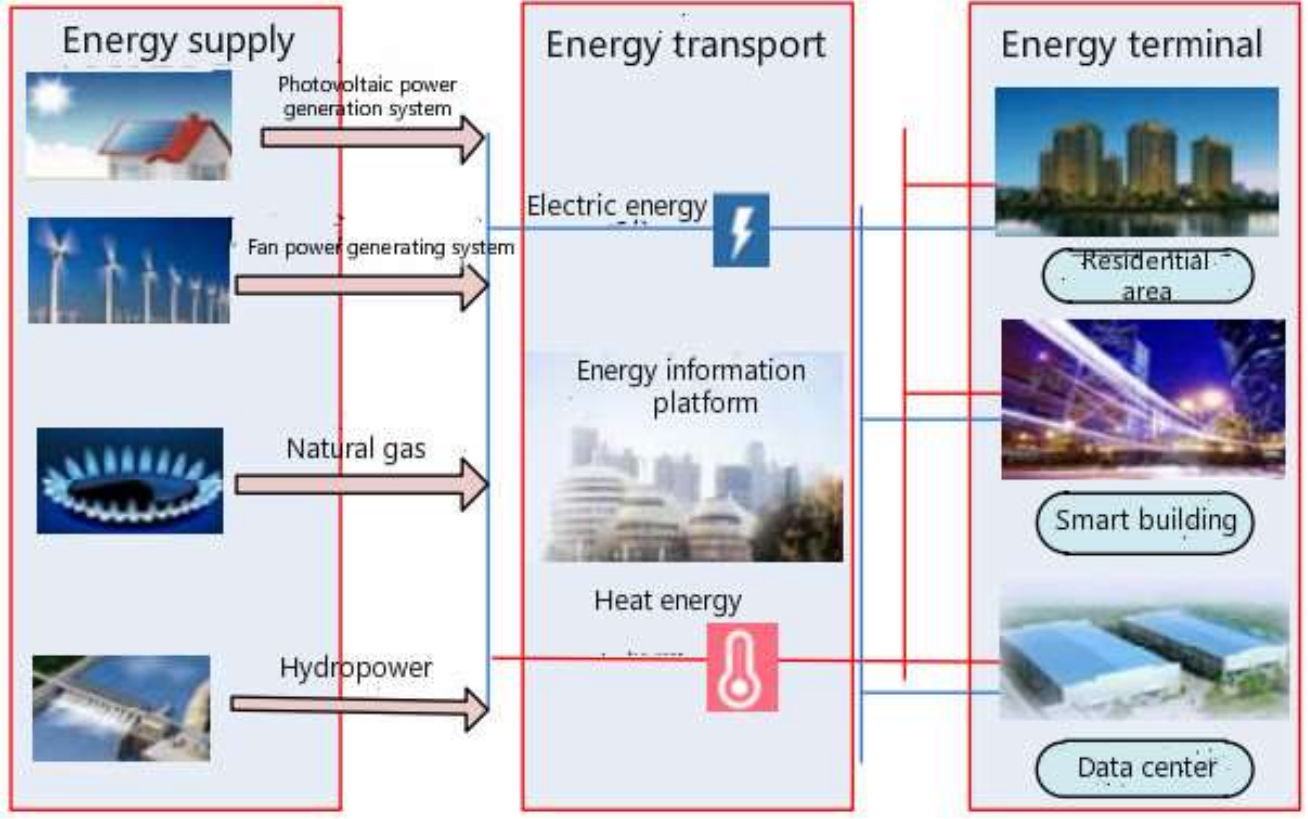

Figure.1 The basic model framework of integrated energy collaborative development

\section{Implementation of Power Distribution Network Integrated Energy Collaborative Optimization Based on Big Data}

Analysis of Integrated Energy Collaborative Development Optimization Algorithm. The goal of this integrated energy collaborative planning is to consider the optimal utilization of resources, minimize the cost of investment and operation of the system, consume the clean energy to the most extent, improve the efficiency of energy utilization and reduce pollution and emission on the basis of ensuring the safe and stable operating conditions of the system. This paper comprehensively considers the construction costs of power grid, CHP and wind-solar hybrid complementary system, as well as the cost of power generation and the penalty cost of abandoning wind and light, the optimization planning mathematical model is set up with the objective of the lowest system comprehensive cost. The objective function expression of the model is as follows:

$$
F=\min \left(\mathrm{f}_{1}+\mathrm{f}_{2}+\mathrm{f}_{3}\right)
$$

Where, $\mathrm{F}$ is the total cost of the regional integrated energy system, F1 is the investment and construction cost in the integrated energy system, F2 is the cost of generating electricity, F3 is the penalty cost of abandoning wind and light and losing load. 


$$
\begin{aligned}
& \mathrm{f}_{1}=k v_{G R I D} \sum_{i=\Phi} u_{i}^{G R I D}+k v_{C H P} \sum_{i=\Phi} u_{i}^{C H P}+k v_{G F} \sum_{i=\Phi} u_{i}^{G F}+k v_{E V} \sum_{i=\Phi} u_{i}^{E V} \\
& f_{2}=\sum_{i \in S_{G}}\left(\alpha_{i}+\beta_{i} \mathrm{P}_{G i}+\delta_{i} \mathrm{P}_{G i}^{2}\right) \\
& f_{3}=p_{\text {penal }, 1} \sum_{i=1}^{T} L_{\text {des }, t}+P_{\text {penal }, 2} \sum_{i=1}^{T} L_{\text {loss }, t}
\end{aligned}
$$

At the same time, all energy resources should meet the following constraint restrictions according to their characteristics:

Considering that the local users are constrained by the upper and lower limit of node voltage, the voltage amplitude must meet the following requirements:

$$
V_{\min } \leq V_{i} \leq V_{\max }
$$

Due to the restriction of feeder current in the planning area, the current amplitude must meet the following requirements:

$$
\left|I_{i}\right| \leq I_{\max }
$$

Due to the restriction of the active power balance in the park, power of regional power grid and distributed power supply output and load power should be balanced:

$$
\sum_{i=1}^{n_{p}} P_{t}=P_{g}+P_{c}=P_{D G}-P_{1},\left(\mathrm{i}=1,2, \ldots, \mathrm{n}_{p}\right)
$$

A Grid Coordinated Planning Strategy of GIS System. The definition of grid is to divide a region into several grid small units according to certain rules, so that we can make more detailed management for the region. The so-called "grid" refers to the space units after the management area or object is divided, which similar to land block and responsibility area and can be big and small. The so-called "net" refers to the appropriate area composed of several grids. In this paper, the concept of grid is introduced into the power distribution network planning. According to the grid structure, load distribution, geographic boundary and other actual situations, the complex power distribution network is divided into several relatively independent grids and the status and existing problems of the grid are analyzed. Combined with typical load forecasting models in big data, load forecasting is made for three periods of near, middle and long term and grid target is formulated. According to the differentiated planning standards, combined with the distribution of regional integrated energy, the primary grid structure planning and integrated energy facilities coordination planning of power distribution network are carried out in sub region. Through the grid planning, the power and energy channel schemes are proposed, the overhead, cable channels required for the road and the distribution path of the integrated energy transport channel are reserved. Combined with urban road construction, we should avoid building and re dismantle, further optimize the resource utilization to achieve the coordinated planning of regional energy and formulate fine coordinated planning strategy. The strategy flow chart is shown in Figure 2. First, the current situation and energy resource conditions of power grid in demonstration area are analyzed, the grid is preliminarily divided through regional characteristics, and the demand for power load and integrated energy development in the grid is analyzed. Secondly, grid partition correction and coordinated planning strategy restriction formulation are carried out. Finally, collaborative planning strategy and benefit evaluation are formulated. Based on the characteristics of the regional development and the power supply requirements of the important user bottom grid, the integrated energy coordination plan is put forward. 


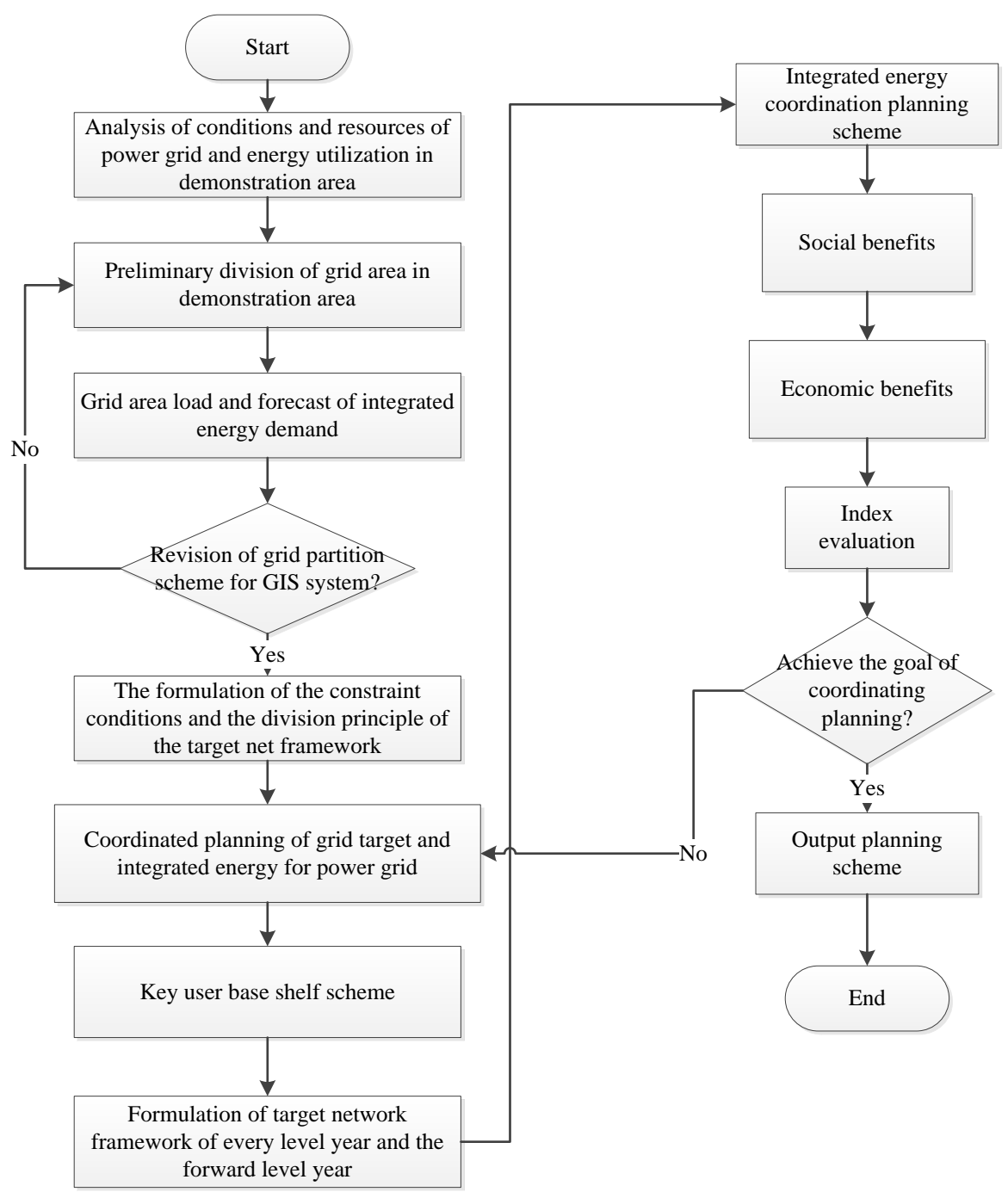

Figure. 2 The planning strategy flow chart

Results and benefit analysis of planning strategy. Through integrated energy coordination planning and energy storage device's peak shaving and valley filling function, according to the requirements of the power grid Corp planning technology principles and considering the demand of the capacity load ratio 1.9 2.1, there are five $110 \mathrm{kV}$ substations in the forward level year new area, and compared with the traditional planning scheme, two $110-\mathrm{kV}$ substations were reduced. The forward level year increases the capacity of 378-MVA and the main transformer capacity is reduced by 378 MVA compared with the traditional scheme. The $110-\mathrm{kV}$ main transformer capacity of the new area in 2030 will reach 837 MVA. Considering the regional integrated energy supply and demand capacity, the whole area is divided into 48 grids and 53 groups of 10 $\mathrm{kV}$ typical connection groups are provided for power supply. The forward level year needs to increase 69 medium voltage lines, and a total of 153 lines of power supply plan. In each grid, the power supply line is more clear and orderly, which is reduced by 43 medium voltage lines compared with the traditional scheme. Park new energy consumptive the forward level year accounted for about $35 \%$, the proportion increased by about $25 \%$ compared with the traditional planning scheme. The planning scheme is shown in Figure 3. 


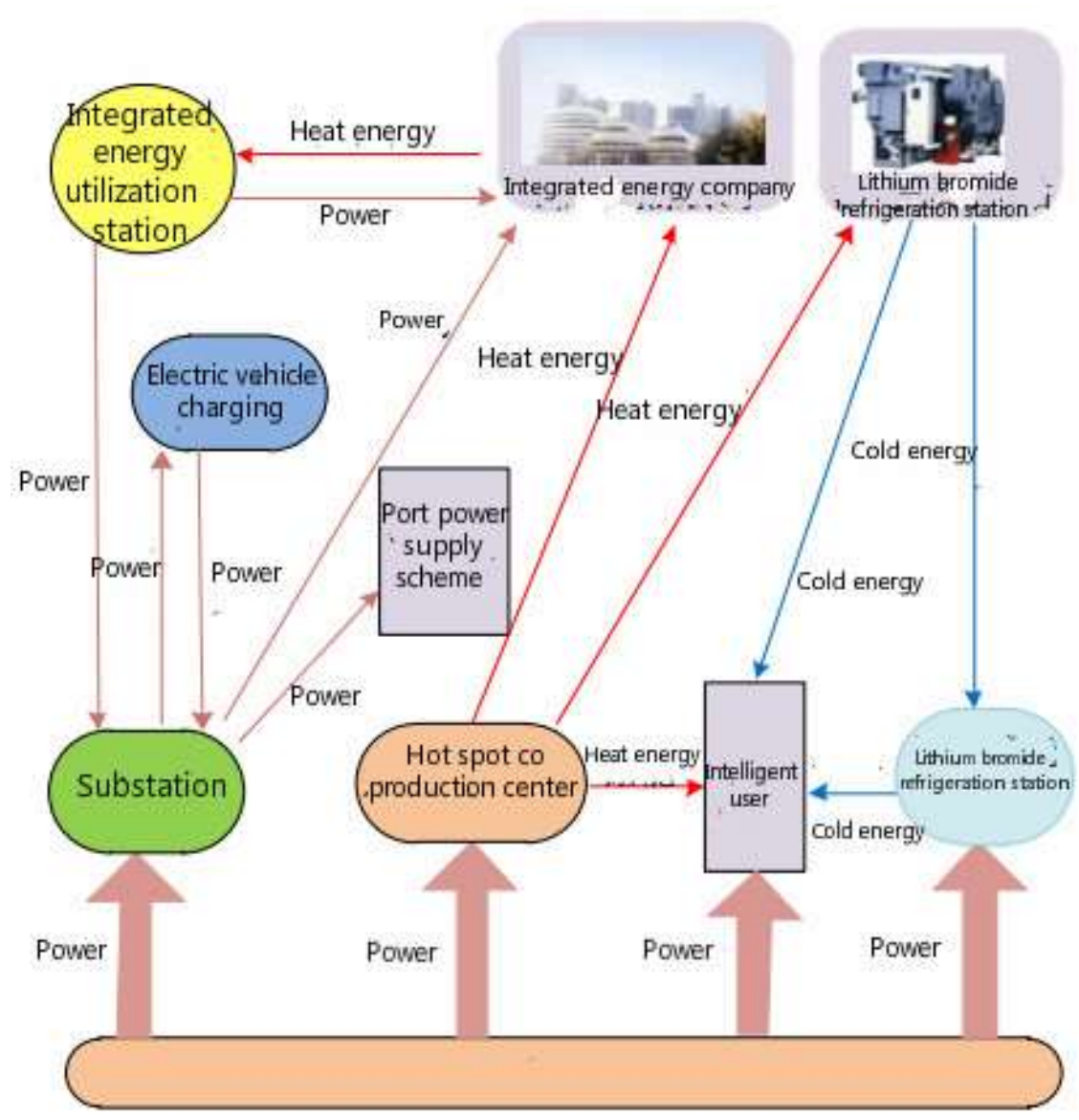

Figure.3 Planning scheme

The comparison of the scheme is shown in Table 1. Compared with the traditional scheme, the scheme in this paper has achieved the goal of integrated energy collaborative development, green energy and low carbon security of energy structure and the greatly improving of energy utilization efficiency.

Table. 1 The comparison of the scheme

\begin{tabular}{cccccc}
\hline Project & $\begin{array}{c}110 \mathrm{kV} \\
\text { substation }\end{array}$ & $\begin{array}{c}\text { New main transformer } \\
\text { capacity /MVA }\end{array}$ & $\begin{array}{c}\text { New medium } \\
\text { voltage line }\end{array}$ & $\begin{array}{c}\text { Percentage of } \\
\text { new energy/\% }\end{array}$ & $\begin{array}{c}\text { Energy } \\
\text { efficiency }\end{array}$ \\
\hline $\begin{array}{c}\text { Traditional } \\
\text { method }\end{array}$ & 7 & 756 & 196 & 10 & Commonly \\
$\begin{array}{c}\text { Proposed } \\
\text { method }\end{array}$ & 5 & 837 & 153 & 35 & Higher \\
\hline
\end{tabular}

\section{Conclusion}

This paper considers the various forms of resources in the energy supply side and demand side should be integrated into a whole to make overall utilization. Based on the construction of big data center and energy information sharing platform, a comprehensive model of multi energy complementary and coordinated development is established. In order to make the planning more precise, we use the gridding method of geographic information system to carry out coordinated planning. Taking the integrated energy system as the center, making full use of the cold, heat, electric triple supply technology, lithium bromide refrigeration technology, wind and solar complementary cooperative power supply scheme, port power supply scheme and electric vehicle charging technology to achieve energy interaction and cascade utilization, which reduces the peak-shaving pressure of the power grid, gives full play to the capacity of the energy storage system and improves the ability to consume the clean energy. 


\section{References}

[1] Cui D, Li X, Ge Y. System design and development of distribution network planning quality indicator monitoring platform[C]// Energy Internet and Energy System Integration. IEEE, 2018:1-6.

[2] Guo Y, Liu J, Wei Z, et al. Load supply capability of distribution network[J]. Electric Power Automation Equipment, 2018.

[3] Wei J, Zhang D, Luo H, et al. Distribution Network Fault Diagnosis Based on Improved RBF Neural Network[J]. Journal of Jishou University, 2018.

[4] Yu L, Zhan F. Research Real-time of Distribution Network Monitoring Web System[J]. Electronics World, 2018.

[5] Farouk A, Tarawneh O, Elhoseny M, et al. Different Architectures of Quantum Key Distribution Network[J]. 2018.

[6] Lou C, Zhang X, Cong P, et al. Service Restoration Strategy of Active Distribution Network with Soft Open Points[J]. Automation of Electric Power Systems, 2018.

[7] Tang W, Zhang H H, Xiao S. Research on Accurate Investment Strategy of Distribution Network Based on Attribute Classification of Projects[J]. Science Technology \& Industry, 2018.

[8] Blaauwbroek N, Kuiken D, Nguyen P, et al. Distribution network monitoring: Interaction between EU legal conditions and state estimation accuracy[J]. Energy Policy, 2018, 115:78-87.

[9] Wei W, Gao H, Zhu J, et al. Evaluation on Sustainable Development Capability of Smart Distribution Network Based on System Dynamics[J]. Proceedings of the CSU-EPSA, 2018.

[10] Park E S. Harmonics Amplification of Switching-in of Capacitors Installed in Electric Distribution Network[J]. Journal of the Korean Institute of Illuminating \& Electrical Installation Engineers, 2018, 32. 